Optical investigation of shallow acceptor states in GaN grown by hydride vapor-phase epitaxy

V. Kirilyuk, P. R. Hageman, P. C. M. Christianen, and P. K. LarsenM. Zielinski

Citation: Appl. Phys. Lett. 79, 4109 (2001); doi: 10.1063/1.1427151

View online: http://dx.doi.org/10.1063/1.1427151

View Table of Contents: http://aip.scitation.org/toc/apl/79/25

Published by the American Institute of Physics

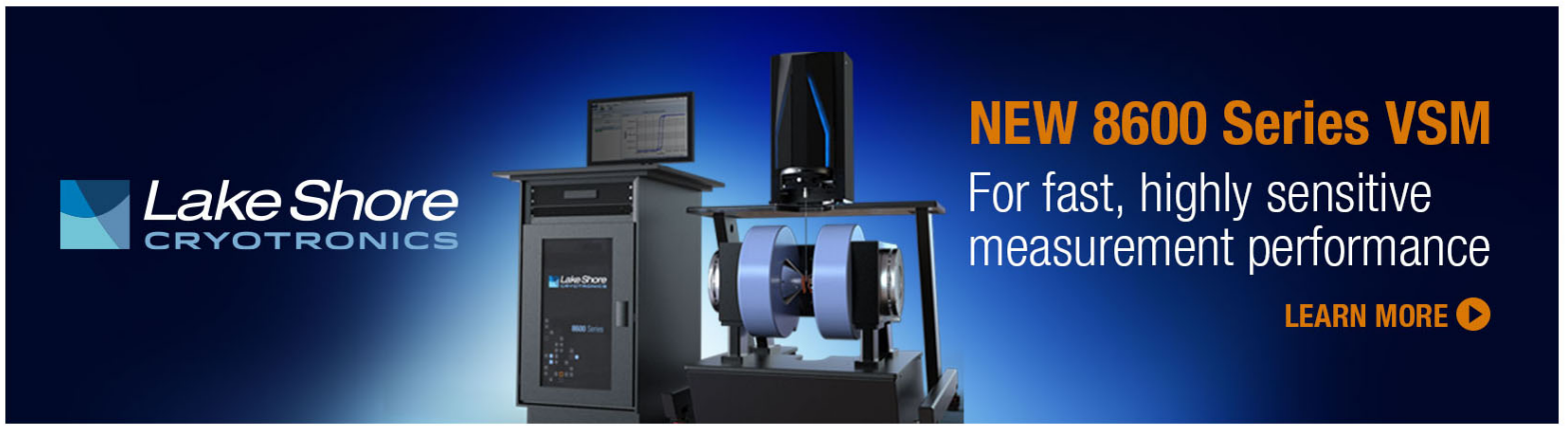




\title{
Optical investigation of shallow acceptor states in GaN grown by hydride vapor-phase epitaxy
}

\author{
V. Kirilyuk, ${ }^{\text {a) }}$ P. R. Hageman, P. C. M. Christianen, and P. K. Larsen \\ Research Institute for Materials, University of Nijmegen, Toernooiveld 1, 6525 ED Nijmegen, \\ The Netherlands \\ M. Zielinski \\ Institute of Physics, Polish Academy of Sciences, Al Lotnikow 32/46, 02-668 Warsaw, Poland
}

(Received 19 June 2001; accepted for publication 10 October 2001)

\begin{abstract}
The evolution of low-temperature photoluminescence (PL) spectra with the thickness of the layer $(3-400 \mu \mathrm{m})$ is investigated on high-quality GaN grown by hydride vapor-phase epitaxy. With increasing layer thickness, three acceptor bound exciton peaks are found to reduce in intensity, although the impurity concentrations, measured by secondary ion mass spectrometry, do not depend on the sample thickness. The observed acceptor transitions are attributed to intrinsic defects, originating from the substrate/layer interface and decreasing in density with the thickness of the layer. The optical properties, studied by reflectance, temperature and excitation power dependent PL, are compared to those of homoepitaxial GaN films grown by metalorganic chemical vapor deposition. () 2001 American Institute of Physics. [DOI: 10.1063/1.1427151]
\end{abstract}

Hydride vapor phase epitaxy (HVPE) has already proved to be well suited for the growth of thick GaN layers, which has led to the fabrication of free-standing GaN substrates for subsequent homoepitaxial growth. ${ }^{1-5}$ Due to the high possible growth rates (up to hundreds of micrometers per hour), the capability of handling large wafer sizes and being relatively inexpensive, the HVPE method is very promising for industrial applications. Despite serious drawbacks such as layer cracking and inhomogeneous free carrier distributions, recently reported GaN layers grown by HVPE show rather low defect densities, ${ }^{2,6}$ narrow photoluminescence (PL) peaks, ${ }^{2,5}$ and high electron mobilities. ${ }^{1,7}$ Therefore, HVPE grown material of high quality can be used to study the thickness dependence of the structural and optical properties in order to clarify often controversial results, obtained on relatively thin, highly stressed, and defective GaN layers grown by metalorganic chemical vapor deposition (MOCVD).

In this letter, we present results of a PL study on several HVPE-grown GaN layers in order to follow the evolution of the free and bound exciton peaks with the thickness of the films. The narrow acceptor-bound exciton lines $(2-3 \mathrm{meV})$ are found to decrease in intensity, and even disappear, as the thickness of the GaN layer increases. Secondary ion mass spectrometry (SIMS) depth profiling shows, however, no changes in the impurity concentrations with varying sample thickness. We, therefore, attribute these shallow acceptor levels to be related to structural defects originating from the film/substrate interface and decreasing in density with film thickness. In addition, we show that similar acceptor-boundexciton transitions are also present in the PL spectra of homoepitaxial MOCVD layers.

Nominally undoped GaN films of thicknesses ranging from 3 to $400 \mu \mathrm{m}$ were deposited in a home built, horizontal

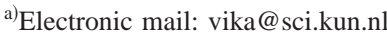

HVPE reactor using MOCVD-grown GaN layers $(\sim 1-2$ $\mu \mathrm{m})$ on sapphire substrates. The HVPE reactor was equipped with a two-zone furnace and operated at atmospheric pressure. Nitrogen was used as a carrier gas $(1 \mathrm{slm})$ and $\mathrm{NH}_{3}$ as a nitrogen precursor $(0.6 \mathrm{slm})$. The reactor was designed to obtain laminar flow and allows mixing of the $\mathrm{NH}_{3}$ and the $\mathrm{GaCl}$ just above the susceptor. The $\mathrm{GaCl}$ growth species were in situ synthesized by passing pure $\mathrm{HCl}$ over liquid gallium $(7 \mathrm{~N})$ at $890^{\circ} \mathrm{C}$. At a growth temperature of $980^{\circ} \mathrm{C}$, the GaN growth rate was varied between 40 and $400 \mu \mathrm{m} / \mathrm{h}$ depending on the amount of $\mathrm{HCl}(5-50 \mathrm{sccm})$.

The free carrier concentration in the thinnest layer $(\sim 3.5$ $\mu \mathrm{m})$ under investigation, as determined by Hall measurements employing the Van der Pauw configuration, was found to be $\sim 6 \times 10^{17} \mathrm{~cm}^{-3}$. High-resolution $\mathrm{x}$-ray diffraction (XRD) measurements were performed on a Bruker D8 instrument. Typical values of the full width of half maximum (FWHM) of a symmetric reflection in the XRD $\omega$ scans are 260-270 and 400-600 arcsec for the 5-8 and 10-400 $\mu \mathrm{m}$ films, respectively. SIMS profiling was performed with a Cameca 6F instrument using $14.5 \mathrm{keV}$ primary $\mathrm{Cs}+$ ions and a negative secondary beam.

Photoluminescence spectra were measured with the 325 $\mathrm{nm}$ line of a $\mathrm{cw} \mathrm{HeCd}$ laser using an excitation power density up to $50 \mathrm{~W} / \mathrm{cm}^{2}$. The PL emission was dispersed by a 0.6-m-single monochromator providing a spectral resolution of about $0.35 \mathrm{meV}$ in the spectral region of interest (3.35$3.55 \mathrm{eV})$. Quasimonochromatic light from a $450 \mathrm{~W}$ Xe lamp, dispersed by a $0.25 \mathrm{~m}$ monochromator (spectral resolution $\sim 1 \mathrm{meV}$ ), was used in reflectance measurements at nearnormal incidence to the sample surface. The low temperature optical spectra were studied using a helium-flow cold-finger cryostat.

Low temperature PL and reflectance spectra of GaN layers of different thicknesses are shown in Fig. 1. Fitting the reflectance data with a set of four oscillators, ${ }^{8}$ we obtain the positions of the free $A\left(X_{A}^{n=1}\right), B\left(X_{B}^{n=1}\right)$, and $C\left(X_{C}^{n=1}\right)$ 


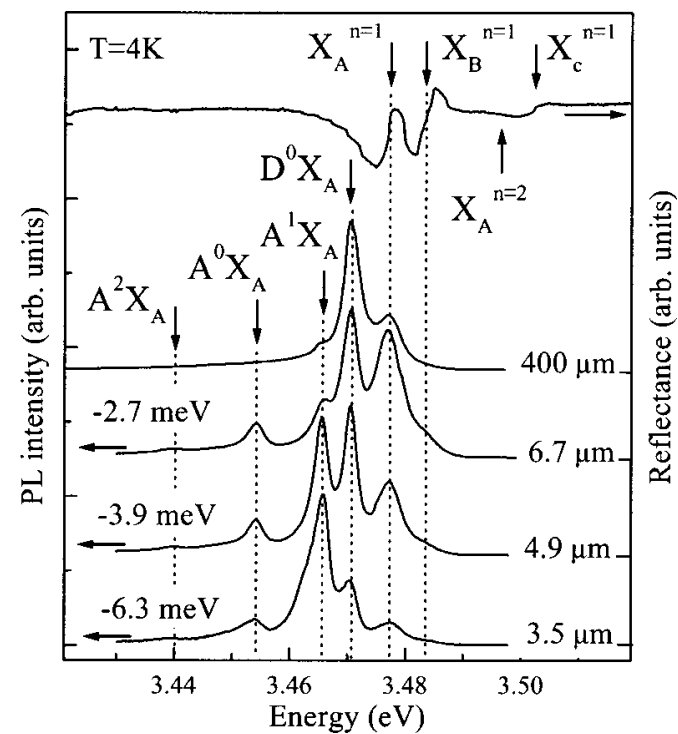

FIG. 1. Low temperature PL and reflectance spectra of HVPE grown GaN of different thicknesses. The numbers on the curves denote the corresponding film thickness. The PL spectra are shifted in order to line up the $X_{A}^{n=1}$ peak (values and directions of shifting are indicated with arrows). The spectra show free $A, B$, and $C$ excitons $\left(X_{A}^{n=1}, X_{B}^{n=1}\right.$, and $X_{C}^{n=1}$, respectively), a first excited state of the $A$-band exciton $\left(X_{A}^{n=2}\right)$, donor bound excitons $\left(D^{0} X_{A}\right)$, and acceptor bound exciton transitions $\left(A^{1} X_{A}, A^{0} X_{A}\right.$, and $\left.A^{2} X_{A}\right)$. The reflectance spectrum is measured on the $4.9-\mu \mathrm{m}$-thick sample and is shifted by $3.9 \mathrm{meV}$ to lower energy.

excitons and the first excited state ( $2 \mathrm{~S}$ state) of the $A$-band exciton (denoted as $X_{A}^{n=2}$ ) for all studied samples. Although the $X_{A}^{n=2}$ transition is weak, it has to be included for a proper description of the full reflectance spectrum. The PL spectra are shifted in order to line up the free $A$-exciton positions taking a freestanding $400 \mu \mathrm{m}$ sample as a reference. All PL spectra can be well fitted using Lorentzian-shaped peaks, revealing several donor and acceptor bound excitons. With increasing film thickness (from 3 to $10 \mu \mathrm{m}$ ), the bound exciton peaks become narrower (the FWHM decreases from 3 to 2.2 $\mathrm{meV}$ ), the $X_{A}^{n=1}$ line becomes more pronounced and all transitions are continuously redshifted, indicating a release of stress and an improvement of the material quality. The intensities of the acceptor-bound exciton lines in the PL spectra decrease with the film thickness and some of them even disappear. The $400-\mu \mathrm{m}$-thick freestanding layer shows slightly broader PL peaks and a relatively low intensity of the $X_{A}^{n=1}$ line. We mainly connect these effects with crack formation in the films (cracking in the HVPE layers sometimes occurs for thicknesses exceeding $10 \mu \mathrm{m}$, depending on the growth rate). In order to further characterize the free and bound exciton transitions and to confirm the identification of all peaks mentioned above, we performed temperature- and excitationdependent PL experiments. PL spectra of the $4.9 \mu \mathrm{m}$ thick layer at temperatures ranging from 4 to $300 \mathrm{~K}$ are presented in Fig. 2, together with the evolution of the phonon replicas of the excitonic lines (inset). The donor bound exciton line $\left(D^{0} X_{A}\right.$ at $\left.E=3.4746 \mathrm{eV}\right)$ is the strongest one at $T=4 \mathrm{~K}$, but starting from $T \sim 30 \mathrm{~K}$ the free $A$-exciton peak dominates the spectrum up to room temperature. From the difference in the energy positions of the $X_{A}^{n=1}(E=3.4812 \mathrm{eV})$ and $X_{A}^{n=2}(E$ $=3.4992 \mathrm{eV}$ ) lines, which are well resolved in the emission spectra at $T=4 \mathrm{~K}$, we calculate the binding energy of the

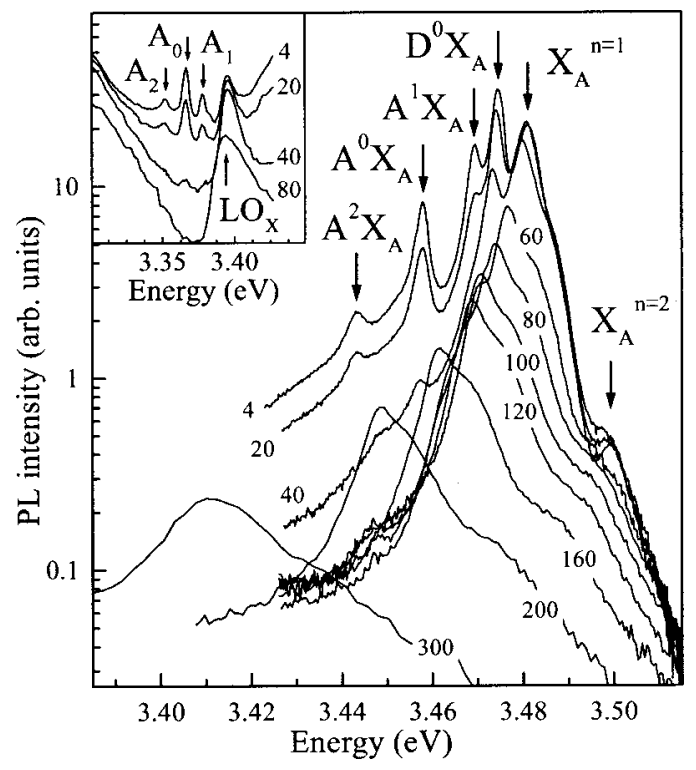

FIG. 2. Temperature-dependent PL spectra (from 4 to $300 \mathrm{~K}$ ) of a $4.9-\mu \mathrm{m}$ thick GaN epilayer grown by HVPE. The numbers on the curves denote the corresponding temperatures in $\mathrm{K}$. The free $A$-exciton transition $\left(X_{A}^{n=1}\right)$ dominates the emission at room temperature. The development of the longitudinal optical (LO) phonon replicas of the free and bound exciton transitions is shown in the inset, where $\mathrm{LO}_{X}, A_{0}, A_{1}$, and $A_{2}$ stand for the LO replicas of the $X_{A}^{n=1}, A^{0} X_{A}, A^{1} X_{A}$, and $A^{2} X_{A}$ transitions, respectively.

free A exciton in a straightforward way using the hydrogen atom model adopted for excitons and obtain $E_{A}^{b}=24$ $\pm 1 \mathrm{meV}$, which is similar to previously reported values. ${ }^{2,8}$ Thermal quenching of the $D^{0} X_{A}$ peak occurs at $\sim 80-100 \mathrm{~K}$ corresponding to the observed localization energy $\left(E_{1}^{D X}\right.$ $=6.6 \mathrm{meV}$ at $T=4 \mathrm{~K}$ ), suggesting that the release of donorbound to free excitons is responsible for the quenching. The identification of three narrow PL lines at energy positions $3.4696\left(A^{1} X_{A}\right), 3.4581\left(A^{0} X_{A}\right)$, and $3.4433 \mathrm{eV}\left(A^{2} X_{A}\right)$ as excitons bound to neutral acceptors is consistent with the observation of their longitudinal optical (LO) phonon replicas (inset Fig. 2), due to a strong coupling of acceptor-bound excitons with LO phonons in polar semiconductors. ${ }^{9,10}$ Thermal quenching of the $A^{1} X_{A}, A^{0} X_{A}, A^{2} X_{A}$ lines and the corresponding $\mathrm{LO}$ replicas $\left(A_{1}, A_{0}, A_{2}\right)$ takes place at $40-60 \mathrm{~K}$. The line labeled $\mathrm{LO}_{X A}$ at $\sim 3.39 \mathrm{eV}$ is a one-phonon assisted emission of the A-band free excitons and this transition dominates in the phonon-assisted-transition region at the elevated temperatures.

To evaluate the concentrations of impurities in our HVPE grown material, we performed SIMS depth profiling and obtained values of about $2-3 \times 10^{17}$ atoms $/ \mathrm{cm}^{3}$ for $\mathrm{Si}$, $\mathrm{O}$, and $\mathrm{C}$ and $\sim 10^{18}$ atoms $/ \mathrm{cm}^{3}$ for $\mathrm{H}$ in the top $1 \mu \mathrm{m}$ region. In fact, these values are 5 to 10 times lower than those measured in our hetero- and homoepitaxial MOCVD layers. ${ }^{11,12}$ Moreover, the impurity concentrations do not depend on the sample thickness. Only in the $0.2-0.5 \mu \mathrm{m}$ thick epilayer/ substrate interface region the concentrations of impurities were found to be slightly higher, as it was previously seen in other types of GaN samples. ${ }^{11,12}$ This result means, that if the bound-exciton peaks would be related to the impurities studied by SIMS, their peak intensity ratio would be similar for films of different thicknesses, since the probing depth of the PL is only a few hundred nanometers and the impurity con- 


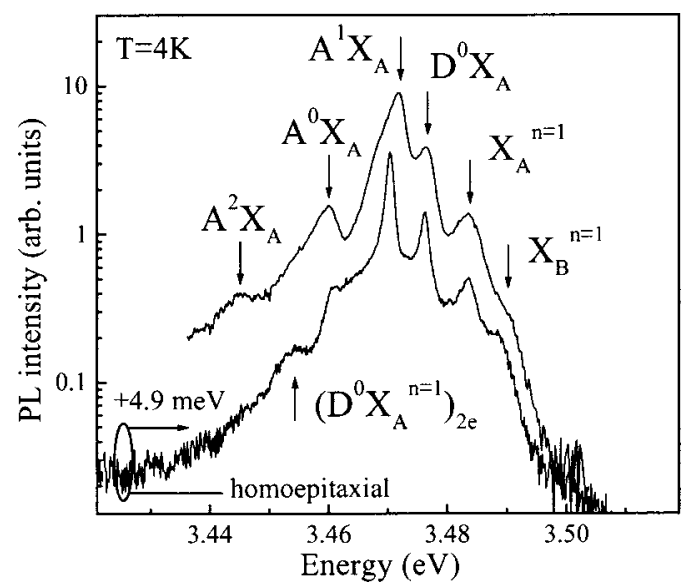

FIG. 3. Comparison of the PL features (at $T=4 \mathrm{~K}$ ) of a $2-\mu$ m-thick homoepitaxial GaN epilayer grown by MOCVD (lower curve) and a 3.5- $\mu \mathrm{m}-$ thick film grown by HVPE (upper curve). The PL spectrum of the homoepitaxial sample is shifted to higher energy to align the free $A$-exciton peaks of both samples. The peak denoted as $\left(D^{0} X_{A}^{n=1}\right)_{2 e}$, is a two-electron replica of the donor bound exciton transition.

centrations in the upper part of all the layers are the same. Therefore, the changes in the relative peak intensities in the epilayers of different thickness (see Fig. 1) suggest that the observed acceptor levels are rather introduced by intrinsic structural defects than by incorporation of impurities. Indeed, recent TEM studies have shown a decrease of dislocation and defect densities with the thickness of HVPE films. ${ }^{13,14}$

This conclusion is further confirmed by comparing the low temperature PL features of the 3.5- $\mu \mathrm{m}$-HVPE layer with those of a $2-\mu \mathrm{m}$-homoepitaxial $\mathrm{GaN}$ layer grown by MOCVD (see Fig. 3), where the latter was shifted by 4.9 $\mathrm{meV}$ to higher energy side in order to align the free A-exciton peaks. In both spectra the acceptor bound excitons $A^{0} X_{A}$ and $A^{1} X_{A}$ are present, although the $A^{1} X_{A}$ line in the thin HVPE film is not a single peak. It consists of at least two optical transitions lying closely to each other, but similar observations have also been made in the PL spectra of several homoepitaxial MOCVD films. ${ }^{15}$ Although the localization energy of these peaks is slightly varying by $\pm 1 \mathrm{meV}$, the temperature and excitation power dependent PL reveals similar behavior of the acceptor-bound-exciton peaks in both types of material. The saturation of the $A^{0} X_{A}$ and $A^{1} X_{A}$ peaks with increasing excitation power takes place in both the HVPE layers and in the homoepitaxial MOCVD films (not shown). We would like to point out, however, that the $A^{0} X_{A}$ and $A^{1} X_{A}$ lines appear in samples with completely different substrate preparations: reactive ion etching, mechanochemical etching or no etching/polishing, as well as using different growth techniques. Since the interface region and the ambient of the reactor are usually the main sources of unintentional impurity incorporation, it is very unlikely that the observed $A^{0} X_{A}^{n=1}$ and $A^{1} X_{A}^{n=1}$ transitions originate from excitons bound to unintentionally incorporated impurities.

In conclusion, the optical investigation of high quality HVPE grown GaN layers shows that most PL features of the HVPE films are essentially similar to those of homoepitaxial layers grown on single-crystal GaN substrates by MOCVD. The development of the PL emission with the thickness of the HVPE films reveals the quenching of acceptor-boundexciton peaks and appears to be independent on impurity concentrations as measured by SIMS. Therefore, the observed acceptor levels are assigned to intrinsic defects originating from the substrate/layer interface and decreasing in density with the thickness of the film.

${ }^{1}$ R. J. Molnar, W. Götz, L. T. Romano, and N. M. Johnson, J. Cryst. Growth 178, 147 (1997).

${ }^{2}$ B. J. Skromme, J. Jayapalan, R. P. Vaudo, and V. M. Phanse, Appl. Phys. Lett. 74, 2358 (1999).

${ }^{3}$ M. K. Kelly, R. P. Vaudo, V. M. Phanse, L. Görgens, O. Ambacher, and M. Stutsmann, Jpn. J. Appl. Phys., Part 2 38, L217 (1999).

${ }^{4}$ E. A. Stach, M. Kelsch, E. C. Nelson, W. S. Wong, T. Sands, and N. W. Cheung, Appl. Phys. Lett. 77, 1819 (2000).

${ }^{5}$ C. R. Miskys, M. K. Kelly, O. Ambacher, G. Martínez-Criado, and M. Stutzmann, Appl. Phys. Lett. 77, 1858 (2000).

${ }^{6}$ P. Visconti, K. M. Jones, M. A. Reshchnikov, F. Yun, R. Cingolani, H. Markoç, S. S. Park, and K. Y. Lee, Appl. Phys. Lett. 77, 3743 (2000).

${ }^{7}$ A. Saxler, D. C. Look, S. Elhamri, J. Sizelove, D. Cull, W. C. Mitchel, M. Callahan, D. Bliss, L. Bouthillette, S.-Q. Wang, C. M. Sung, S. S. Park, and K. Y. Lee, Mater. Res. Soc. Symp. Proc. (accepted for publication).

${ }^{8}$ K. Kornitzer, T. Ebner, K. Thonke, R. Sauer, C. Kirchner, V. Schwegler, M. Kamp, M. Leszczynski, I. Grzegory, and S. Porowski, Phys. Rev. B 60, 1471 (1999); D. G. Chtchekine, Z. C. Feng, S. J. Chua, and G. D. Gilliland, ibid. 63, 125211 (2001).

${ }^{9} \mathrm{~S}$. Permogorov, in Modern Problems in Condensed Matter Science, edited by E. I. Rashba and M. D. Sturge (North-Holland, Amsterdam, 1982), Vol. 2, p. 177.

${ }^{10}$ J. Jayapalan, B. J. Skromme, R. P. Vaudo, and V. M. Phanse, Appl. Phys. Lett. 73, 1188 (1998).

${ }^{11}$ V. Kirilyuk, M. Zielinski, P. C. M. Christianen, A. R. A. Zauner, J. L. Weyher, P. R. Hageman, and P. K. Larsen, Mater. Res. Soc. Symp. Proc. (accepted for publication).

${ }^{12}$ P. J. L. Weyher, A. R. A. Zauner, P. D. Brown, F. Karouta, A. Barcz, M. Wojdak, and S. Porowski, Phys. Status Solidi A 176, 573 (1999).

${ }^{13}$ Z.-Q. Fang, D. C. Look, J. Jasinski, M. Benemara, Z. Liliental-Weber, and R. J. Molnar, Appl. Phys. Lett. 78, 332 (2001).

${ }^{14}$ E. M. Goldys, T. Paskova, I. G. Ivanov, B. Arnaudov, and B. Monemar, Appl. Phys. Lett. 73, 3583 (1998).

${ }^{15}$ V. Kirilyuk, A. R. A. Zauner, P. C. M. Christianen, J. L. Weyher, P. R. Hageman, and P. K. Larsen, Appl. Phys. Lett. 76, 2355 (2000). 